\title{
THE MERCURY-ION OPTICAL CLOCK: TOWARDS A MEASUREMENT OF THE QUADRUPOLE SHIFT
}

\author{
W. H. Oskay", A. Bartels, S.A. Diddams, C.W. Oates, G. Wilpers, L. Hollberg, \\ W. M. Itano, C.E. Tanner ${ }^{\dagger}$, and J. C. Bergquist
}

Time and Frequency Division, National Institute of Standards and Technology

325 Broadway M.S. 847, Boulder, CO 80305 USA

*Fax: (303) 497-7375, Electronic mail: oskay@boulder.nist.gov

†Permanent address: Department of Physics, University of Notre Dame

Notre Dame Indiana 46556 USA

Keywords: optical frequency standards, quadrupole shift

\begin{abstract}
An optical clock based upon a single mercury ion in a radiofrequency trap has the potential for both high stability and high accuracy. The systematic uncertainty of this frequency standard is limited by contributions due to two types of external fields. The clock has previously been operated with a magnetic bias field that destabilizes dark states that limit the rates of laser cooling and state detection. This bias field has previously resulted in a substantial second-order Zeeman shift. We now destabilize the dark states with a polarizationscrambling technique that allows us to operate with only a minimal magnetic field. The second uncertainty that we address here is the magnitude of the quadrupole shift due to the interaction of stray electric-field gradients with the quadrupole moment of the ion. We describe the steps that we are taking to evaluate this shift in the near future.
\end{abstract}

\section{Introduction}

The search for more accurate and stable frequency standards has led to the development of trapped-ion optical clocks. In these systems, the ion trap provides a minimally perturbative environment that allows for high accuracy. Simultaneously, the high frequency of the clock transition, together with its narrow linewidth, leads to stability that makes it possible to realize this accuracy within a practical amount of averaging time.

The mercury-ion frequency standard has been described in detail elsewhere $[6,8]$, and only the most important features are summarized here. In our system, a single ${ }^{199} \mathrm{Hg}^{+}$ion is confined in a cryogenic, spherical, radio-frequency (Paul) trap and is cooled on the ${ }^{2} S_{1 / 2}-{ }^{2} P_{1 / 2}$ dipole transition at 194 nm. The electric-quadrupole ${ }^{2} S_{1 / 2}(F=0)-{ }^{2} D_{5 / 2}\left(F=2, m_{F}=0\right)$ transition at $282 \mathrm{~nm}$ serves as the reference for the frequency standard. This transition is probed by the frequency-doubled output of a highly stabilized dye laser operating at $563 \mathrm{~nm}$. The short-term stability of the laser derives from an isolated, high-finesse, Fabry-Perot cavity, and the fractional instability of this local oscillator is below $510^{-16}$ (corresponding to a laser linewidth under $1 \mathrm{~Hz}$ at $282 \mathrm{~nm}$ ) for times between $1 \mathrm{~s}$ and $10 \mathrm{~s}$. The long-term stability derives from the ion, and a digital servo loop steers the laser frequency to the atomic resonance over longer time scales.

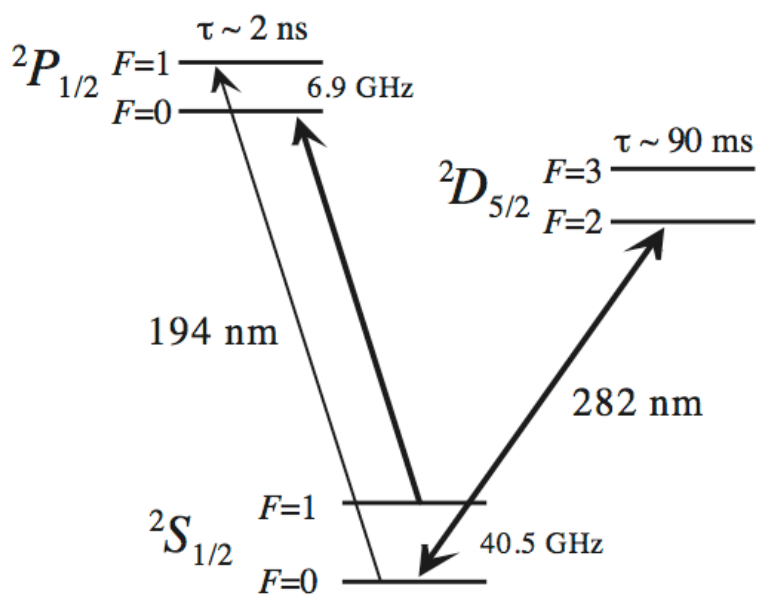

Figure 1: Partial term diagram of the ${ }^{199} \mathrm{Hg}^{+}$ion. The ${ }^{2} S_{1 / 2}-$ ${ }^{2} P_{1 / 2}$ dipole transition at $194 \mathrm{~nm}$ is used for cooling and state detection, while the ${ }^{2} S_{1 / 2}(F=0)-{ }^{2} D_{5 / 2}\left(F=2, m_{F}=0\right)$ "clock" transition at $282 \mathrm{~nm}$ serves as the reference for the optical frequency standard.

The stable light from this optical frequency standard at frequency $f_{\mathrm{Hg}} / 2$ can be phase-coherently compared with signals from other frequency standards by means of a broadband optical frequency comb $[8,11]$. The comb is the output of a broadband femtosecond Ti:Sapphire laser [2], which is further broadened spectrally in a short segment of microstructure fiber. The resulting spectrum consists of a series of spectral lines at frequencies $f_{\mathrm{n}}=n f_{\mathrm{r}}+f_{0}$ for integer $n$, which are spaced by the repetition rate $f_{\mathrm{r}}$ of the laser, near 1 GHz. Both $f_{\mathrm{r}}$ and the offset frequency $f_{0}$ common to all modes can be locked to be rational multiples of $f_{\mathrm{Hg}}$, so that the entire set of spectral lines is phase coherent with $f_{\mathrm{Hg}}$. The 
broadened spectrum typically covers a wavelength range of roughly $520 \mathrm{~nm}$ to $1100 \mathrm{~nm}$ and allows us to compare against other optical frequency standards in that range, such as the calcium optical standard at $657 \mathrm{~nm}[8,9]$. The repetition rate $f_{\mathrm{r}}$ is measured directly with a photodiode to produce a microwave-frequency output at $1 \mathrm{GHz}$ or its harmonics that can be used for comparisons with frequency standards at lower frequencies. As an example, recent comparisons against a calibrated reference signal from a hydrogen maser in the NIST atomic time scale have led to constraints on the present-day variation of the fine structure constant [6].

In the past year, we have moved the clock system into an improved laboratory and resumed progress towards evaluating the uncertainties of the system. We now summarize the current status of the system and describe our recent progress towards these evaluations.

\section{Destabilization of Dark States}

The ${ }^{2} S_{1 / 2}-{ }^{2} P_{1 / 2}$ transition at $194 \mathrm{~nm}$ is used for cooling the ion and also for determining whether or not the $282 \mathrm{~nm}$ probe pulse has driven the ion to the ${ }^{2} D_{5 / 2}$ metastable state, which has a lifetime of about $90 \mathrm{~ms}$. We begin our experimental cycle by cooling the ion with light tuned $35 \mathrm{MHz}$ to the red of the ${ }^{2} S_{1 / 2}(F=1)-{ }^{2} P_{1 / 2}(F=0)$ transition. A second component of the $194 \mathrm{~nm}$ light is phase-locked $47 \mathrm{GHz}$ to the blue of the main beam, near the resonance with the ${ }^{2} S_{1 / 2}(F=0)-{ }^{2} P_{1 / 2}$ $(F=1)$ transition, and serves as a repumper. After cooling the ion for $3 \mathrm{~ms}$, the repumper is turned off for $15 \mathrm{~ms}$ to pump the ion into the ${ }^{2} S_{1 / 2}(F=0)$ state. We then extinguish the $194 \mathrm{~nm}$ light with a mechanical shutter and expose the ion to the probe light at $282 \mathrm{~nm}$ for $40 \mathrm{~ms}$. Finally, we detect the state of the ion with the method of electron shelving [3,7]. Both components of the $194 \mathrm{~nm}$ light are then turned on, and the absence or presence of light scattered from the ion indicates that we have, or have not, made the transition to the metastable state.

For this sequence of events to be successful, it is important that the total scattering rate of the light at $194 \mathrm{~nm}$ is high enough that we can unambiguously determine the state of the ion after the probe period. When the ion is excited to the ${ }^{2} P_{1 / 2}$ state, it usually decays rapidly to the ${ }^{2} S_{1 / 2}$ ground state. The ${ }^{2} P_{1 / 2}$ state is short-lived, with a lifetime about 2.9 ns [12]. However there are several factors that can reduce the fluorescence rate. First, off-resonant excitation can lead to the atom decaying to the "wrong" hyperfine ground state, for which we have the repumper described earlier. The ion will also occasionally decay instead to one of two metastable states $\left({ }^{2} D_{5 / 2}\right.$ or $\left.{ }^{2} D_{3 / 2}\right)$ that temporarily inhibit fluorescence.

A more important concern is that even if the ion does decay into the ${ }^{2} S_{1 / 2}(F=1)$ state, the ion can still be pumped into dark states where it does not fluoresce. Generally speaking, an atom with a $J=1$ to $J^{\prime}=0$ transition will become coherently trapped in a dark state if exposed to light of constant polarization [3]. As this happens, the fluorescence rate approaches zero, so it is critically important to destabilize these dark states. One solution is to split the atomic energy levels, usually with a magnetic field. A second solution is to modulate the polarization of the light that is causing the atom to fluoresce [5].

Previously in the operation of the mercury-ion optical clock, we have applied a magnetic bias field of typical value $\mathrm{B}=0.3$ $\mathrm{mT}$ to destabilize the dark states. This field strength has proven sufficient to allow us to count the scattered photons on a photomultiplier tube with a detected rate of over $10 \mathrm{kHz}$. This rate is much lower than the scattering rate of the ion (due to various inefficiencies in the imaging system), however it is more than enough fluorescence to operate the clock. The disadvantage of such a strong magnetic field is the perturbation to the frequency of the clock transition. While the ${ }^{2} S_{1 / 2}(F=0)-{ }^{2} D_{5 / 2}\left(F=2, m_{F}=0\right)$ transition is magnetic-field insensitive to first order, there is a quadratic Zeeman shift $\Delta f=k^{(2)} B^{2}$ that must be taken into account. The magnitude of the shift is $1.7 \mathrm{kHz}$ for $\mathrm{B}=0.3 \mathrm{mT}$, where the typical fluctuations in our unshielded environment lead to an uncertainty of $2.2 \mathrm{~Hz}$. The uncertainty in the coefficient $k^{(2)}=$ $-18.925(28) \mathrm{kHz} / \mathrm{mT}^{2}$ [10] leads to an additional uncertainty of $0.5 \mathrm{~Hz}$ on the clock transition frequency. Since the magnitude of the shift varies as $B^{2}$, going to a lower field makes the system less sensitive to small fluctuations in the ambient field.

We have now implemented a system of polarization modulation to destabilize the dark states so that we can operate with much lower magnetic fields. In order to fully avoid the dark states, it is necessary to have polarization components in three orthogonal directions, each with a different time dependence. The simplest way to implement this is to have two noncollinear beams intersecting the ion, where one of the two beams has its polarization modulated as a function of time. On the ion trap table, the $194 \mathrm{~nm}$ light is divided evenly into two beams. The first beam has linear polarization and travels in the horizontal plane to intersect the ion in the center of the rf trap. The second beam passes through an electro-optic modulator (EOM) that modulates the polarization. After the modulator, the beam is reflected off a mirror positioned below the ion trap and onto the ion. The propagation axis of the second beam is 40 degrees away from the first beam in the horizontal plane, and it is oriented 35 degrees from vertical. The polarization vector of the first beam is oriented to be in the plane spanned by the two beams. A similar system was previously implemented (using a photoelastic modulator) for a microwave mercury-ion frequency standard based upon the ground-state hyperfine transition [5].

The EOM is a transverse electro-optic phase modulator made from potassium dideuteride phosphate $(\mathrm{KD} * \mathrm{P})$. It is oriented with respect to the incident linear light polarization such that it acts as a polarization rotator. Let us write the electric field of the incident light as $\mathbf{E}_{\text {in }}(t)=\mathrm{E}_{\text {in }} \boldsymbol{x} \cos (\omega t)$, where $\boldsymbol{x}$ is a unit vector orthogonal to the propagation direction $\boldsymbol{k}$. Under 
sinusoidal driving at frequency $\omega_{\mathrm{m}}$, the output electric field is described by

$$
\begin{gathered}
\mathbf{E}_{\text {out }}(t)=\eta \mathrm{E}_{\text {in }}\left[x \cos (\omega t) \cos \left(\Delta \sin \left(\omega_{\mathrm{m}} t\right)\right)+\right. \\
\left.y \sin (\omega t) \sin \left(\Delta \sin \left(\omega_{\mathrm{m}} t\right)\right)\right] .
\end{gathered}
$$

Here, $\eta$ is a constant and $\boldsymbol{y}$ is the unit vector orthogonal to $\boldsymbol{x}$ and $\boldsymbol{k}$. The sinusoidal modulation causes the polarization to rotate back and forth through linear, left and right elliptical, and left and right circular. The modulation index $\Delta$ must be chosen at a value high enough that there is a significant component in both the $\boldsymbol{x}$ and $\boldsymbol{y}$ directions, yet low enough that the laser power can be predominantly on the red side of the atomic resonance. The driving frequency $\omega_{\mathrm{m}}$ is likewise chosen between extremes where the drive is either too slow to destabilize the dark state or high enough that there is little laser power near the atomic resonance. The parameters were chosen to maximise the final fluorescence rate, based on computational work by Berkeland and Boshier [4]. For our system, the modulation index has a value near $\Delta=7$, and the drive frequency $\omega_{\mathrm{m}}$ is near $2 \pi \times 4.7 \mathrm{MHz}$. The EOM is driven through a helical resonator attached to the EOM. The combined system of the resonator and modulator has a quality factor of approximately 230 and is directly driven by a frequency synthesizer with $20 \mathrm{dBm}$ of output power.

Using this polarization modulation system, we have been able to achieve typical fluorescence count rates in excess of 6 $\mathrm{kHz}$, even with vanishing magnetic fields. It is still convenient to operate with a weak magnetic bias field that defines the quantization axis and splits apart the magnetic sublevels of the ${ }^{2} D_{5 / 2}(F=2)$ state. We determine the magnetic field magnitude by measuring the linear Zeeman splitting between the clock transition ${ }^{2} S_{1 / 2}(F=0)-{ }^{2} D_{5 / 2}\left(F=2, m_{F}=0\right)$ and an auxiliary transition to the ${ }^{2} D_{5 / 2}\left(F=2, m_{F}=2\right)$ state. With a bias field of $0.020 \mathrm{mT}$, the total quadratic Zeeman shift is $-7.6 \mathrm{~Hz}$, and typical fluctuations in the laboratory field lead to an uncertainty of $0.15 \mathrm{~Hz}$ on the clock transition frequency that can be further reduced with magnetic shielding. The uncertainty in the Zeeman coefficient $k^{(2)}$ contributes an additional uncertainty of about $3 \mathrm{mHz}$ to the clock frequency, which could be reduced by measuring the coefficient carefully.

\section{Progress Towards a Measurement of the Quadrupole Shift}

The dominant contribution to the uncertainty budget of the mercury-ion optical clock is presently the quadrupole shift of the ${ }^{2} D_{5 / 2}$ state [6]. In an ideal spherical $\mathrm{rf}$ trap, there are no static electric fields applied. The trap consists of a ring electrode symmetrically driven with rf about ground potential, and two grounded endcap electrodes. In this case there are no static electric field gradients and the clock transition frequency is not a function of the quantization axis orientation. In practice, there are static electric patch charges on the electrodes that we compensate with low voltages applied to the endcaps and with higher potentials applied to separate electrodes in the vicinity of the trap. The quadrupole shift is due to the interaction of the atomic electric quadrupole moment of the ion in the ${ }^{2} D_{5 / 2}$ state with the electric field gradients arising from these patch potentials. The total magnitude of the shift is expected to be less than or of order 1 $\mathrm{Hz}$ [10]. However, this estimate was based upon a HartreeFock calculation and it is possible that there are large deviations from this value $[14,16]$. A series of measurements conducted over several years with several different ion trap surfaces does not show any large variations and is consistent with this low value of the shift. However, we have not yet performed a full evaluation of this shift and so have placed a conservative $10 \mathrm{~Hz}$ error bar on the magnitude of this shift in the absence of this evaluation.

We are now preparing to measure the quadrupole shift. It has been shown that the quadrupole shift will average to zero if the clock transition frequency is measured over three orthogonal quantization axes [10]. In the laboratory, we are planning to orient the magnetic field at the site of the ion trap in thee orthogonal directions. The three magnet coil pairs around the ion trap have been calibrated via spectroscopy on the Zeeman-sensitive components of the ${ }^{2} S_{1 / 2}(F=0)-{ }^{2} D_{5 / 2}$ $(F=2)$ transition. With this calibration, magnetic fields of approximately equal magnitude but near-orthogonal orientation can be quickly exchanged by changing the currents on the magnet coil power supplies. A measurement using this method was previously performed with mercury ions in a linear rf trap, which relied upon static electric fields for confinement [13].

In order to perform this measurement, it is essential that there is a stable "flywheel" that can provide a link between successive measurements of the clock transition frequency. We would like to be able to measure the frequency at each of the three field orientations with imprecision well below $1 \mathrm{~Hz}$. The best flywheel for this measurement would be a second mercury-ion optical standard, which we expect to use for this type of comparison in the long term. In the immediate future, we plan to compare against an optical frequency standard based upon neutral calcium. Figure 2 shows a plot of the Allan deviation [1] between the mercury and calcium standards, as measured with the femtosecond frequency comb. The instability averages to well below $10^{-15}(1 \mathrm{~Hz}$ at the $1.064 \mathrm{PHz} \mathrm{Hg}^{+}$transition frequency) with an averaging period of $1000 \mathrm{~s}$.

In addition to measuring the quadrupole shift due to the stray fields in the trap, we also plan to measure the quadrupole moment by applying a known electric-field gradient to the ion. This can be achieved by symmetrically biasing the two endcap electrodes of the trap several volts above ground. A similar method has recently been employed at the PTB to measure the quadrupole moment in a $\mathrm{Yb}^{+}$ion trap [15]. Since it is possible to rapidly change the bias potential on the endcap electrodes, it may be possible to perform this particular measurement without using any secondary atomic references. The stable high-finesse cavities, which have a predictable linear drift for time scales under $30 \mathrm{~s}$, could act as 
the flywheel for measurements that are conducted on a similar time scale.

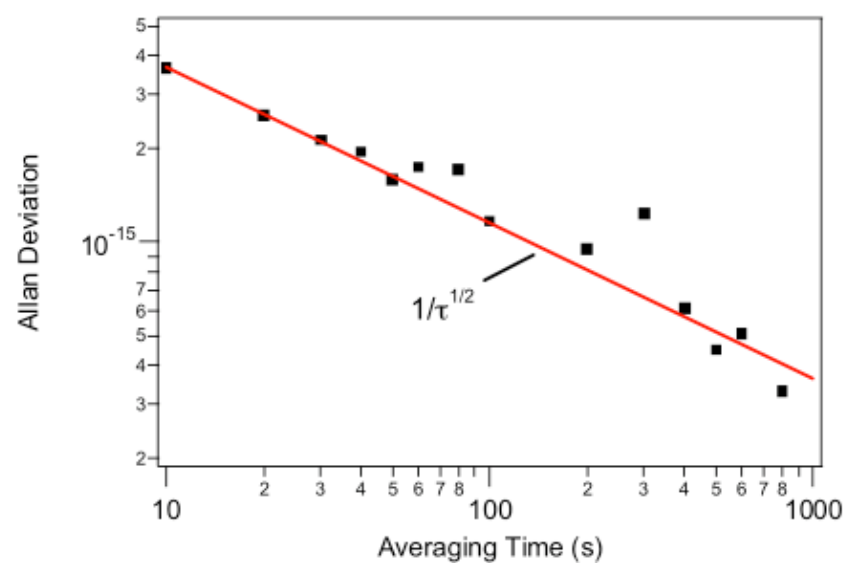

Figure 2: This Allan deviation plot shows the relative stability of the calcium and mercury optical standards as a function of the averaging period $\tau$. This measurement is conducted by counting the effective beat frequency between the two laser sources at $657 \mathrm{~nm}$ and $563 \mathrm{~nm}$ by means of the femtosecond comb system.

\section{Acknowledgements}

We thank Roee Ozeri and Vladislav Gerginov for helpful comments in the preparation of this paper. This work was supported by the Office of Naval Research. C.E.T. was also supported by DOE and NSF. This work of an agency of the U.S. government is not subject to U.S. copyright.

\section{References}

[1] D. W. Allan, "Statistics of Atomic Frequency Standards", Proceedings of the IEEE 54, pp. 221-230 (1966)

[2] A. Bartels and H. Kurz, "Generation of a broadband continuum by a Ti:sapphire femtosecond oscillator with a 1GHz repetition rate", Opt. Lett. 27, pp 1839-1841 (2002).

[3] J. C. Bergquist, W. M. Itano, and D. J. Wineland, "Recoilless optical absorption and doppler sidebands of a single trapped ion", Phys. Rev. A 36, p. 428 (1987).

[4] D. J. Berkeland and M. G. Boshier, "Destabilization of dark states and optical spectroscopy in Zeeman-degenerate atomic systems", Phys. Rev. A 65, 033413 (2002).

[5] D. J. Berkeland, J. D. Miller, J. C. Bergquist, W. M. Itano, and D. J. Wineland, "Laser-Cooled Mercury-Ion Frequency Standard”, Phys Rev. Lett. 80, p. 2089 (1998).

[6] S. Bize, S. A. Diddams, U. Tanaka, C. E. Tanner, W. H. Oskay, R. E. Drullinger, T. E. Parker, T. P. Heavner, S. R.
Jefferts, L. Hollberg,W. M. Itano, and J. C. Bergquist, "Testing the stability of fundamental constants with the ${ }^{199} \mathrm{Hg}^{+}$ single-ion optical clock" Phys. Rev. Lett. 90, 150802 (2003).

[7] H. Dehmelt, "Proposed $10^{14} \delta v<v$ laser spectroscopy on $\mathrm{Tl}^{+}$mono-ion oscillator II (spontaneous quantum jumps)" Bull. Am. Phys. Soc. 20, p. 60 (1975).

[8] S. A. Diddams, Th. Udem, J. C. Bergquist, E. A. Curtis, R. E. Drullinger, L. Hollberg, W. M. Itano, W. D. Lee, C. W. Oates, K. R. Vogel, and D. J. Wineland, "An optical clock based on a single trapped ${ }^{199} \mathrm{Hg}^{+}$ion", Science 293, p. 825 (2001).

[9] J. Helmcke, G. Wilpers, T. Binnewies, C. Degenhardt, U. Sterr, H. Schnatz and F. Riehle, "Optical Frequency Standard Based on Cold Ca Atoms", IEEE Transactions on Instrumentation and Measurement, 52, pp. 250-254, (2003).

[10] Wayne M. Itano, "External-field shifts of the ${ }^{199} \mathrm{Hg}^{+}$ optical frequency standard", J. Res. Natl. Inst. Stand. Technol. 105, p. 829 (2000).

[11] D. J. Jones, S. A. Diddams, J. K. Ranka, A. Stentz, R. S. Windeler, J. L. Hall, and S. T. Cundiff, "Carrier-envelope phase control of femtosecond mode-locked lasers and direct optical frequency synthesis”, Science 288, p. 635 (2000).

[12] E. H. Pinnington, W. Ansbacher, J. A. Kernahan, T. Ahmad, and Z.-Q. Ge, "Lifetime measurements for low-lying levels in $\mathrm{Hg} \mathrm{I}$ and $\mathrm{Hg}$ II using the beam-foil technique", Can. J. Phys. 66, pp. 960-962, (1988).

[13] R. J. Rafac, B.C. Young, J. A. Beall, W. M. Itano, D. J. Wineland, and J. C. Bergquist, "Sub-dekahertz Ultraviolet Spectroscopy of ${ }^{199} \mathrm{Hg}^{+}$", Phys Rev. Lett. 85, p. 2462 (2000).

[14] P. G. H. Sandars and A. J. Stewart, "Beam Resonance measurements of atomic quadrupole moments", in Atomic Physics 3, edited by S. J. Smith and G. K. Walters (Plenum, New York) p. 429-434 (1973).

[15] T. Schneider, Chr. Tamm, E. Peik, "Comparison of Two Single-Ion Optical Frequency Standards at the Hertz Level", Proceedings of the 2003 International Frequency Control Symposium and PDA Exhibition Jointly with the $17^{\text {th }}$ European Frequency and Time Forum, p. 72, (2003).

[16] R. M. Sternhemer, "Atomic quadrupole moments of the excited states of the rare gases", Phys. Rev. A 7, pp. 887-891 (1973). 